\title{
MECHANISM OF THE AMINOLYSIS OF METHYL BENZOATE: A
}

\section{COMPUTATIONAL STUDY}

\author{
Boris Galabov ${ }^{1}$, Yasen Atanasov ${ }^{1}$, Sonia Ilieva ${ }^{1}$, and Henry F. Schaefer III ${ }^{2 *}$ \\ ${ }^{1}$ Department of Chemistry, University of Sofia, Sofia 1164, Bulgaria; \\ ${ }^{2}$ Center for Computational Chemistry, University of Georgia, Athens, GA 30602, U. S. A.
}

\section{Table of contents}

Cartesian coordinates and energies in Hartree for HF/6-31G(d), B3LYP/6-31G(d), B3LYP/6-31+G(d,p) and B3LYP/cc-pVTZ fully optimized geometries of the transition states and intermediates along the concerted and neutral stepwise mechanism of uncatalized and catalyzed ammonolysis of methyl benzoate:

Uncatalyzed ammonolysis of methyl benzoate S2

$\begin{array}{ll}\text { CTS } & \text { S2 } \\ \text { TS1 } & \text { S3 } \\ \text { I1 } & \text { S4 } \\ \text { TS2 } & \text { S5 }\end{array}$

Catalyzed ammonolysis of methyl benzoate CTS $^{\text {catal }} \quad$ S6

$\mathrm{TS}^{\text {catal }} \quad \mathrm{S} 6$

$\mathrm{TS}^{\text {catal }} \quad \mathrm{S} 6$ 


\section{Uncatalyzed ammonolysis of methyl benzoate}

\section{CTS - HF/6-31G(d)}

$C, 0,-0.429603723,0.2462180505,0.0973892041$ C, $\odot,-1.3289385612,1.1596314322,-0.4357085819$ C, $0,-2.6534073304,0.7956679177,-0.6379467151$ C, $0,-3.0786056535,-0.4801888212,-0.3070617559$ C, $0,-2.1769246135,-1.3981767602,0.2180506707$ C $, 0,-0.8545458074,-1.0405329265,0.4167917409$ $\mathrm{C}, \odot, \odot .965253785, \odot .7385521702,0.3382274047$ $0,0,1.4456468416,1.7029351372,-0.1721080758$ $\mathrm{H}, \odot,-0.9853969726,2.1436249056,-\odot .6943835969$ $\mathrm{H}, 0,-3.3452226137,1.5061697899,-1.053661755$ $\mathrm{H}, 0,-4.103897702,-0.7644546881,-0.4652463103$ $\mathrm{H}, 0,-2.5003680881,-2.3962926068,0.4537776373$ $\mathrm{H}, \odot,-\odot .1424711331,-1.765729692, \odot .7618843859$ $0,0,2.0056564137,-0.9151464363,-0.0966350868$ C, $0,2.7031010194,-0.9588929588,-1.2828310349$ $\mathrm{H}, 0,3.6819588277,-1.4256385118,-1.1539052927$ $\mathrm{H}, 0,2.8723448164,0.0436866517,-1.6859016438$ $\mathrm{H}, 0,2.1672084938,-1.5341408089,-2.0412440897$ $\mathrm{N}, 0,1.5397448296,0.3830674676,1.6981309993$ $\mathrm{H}, 0,0.8733519168,0.0558400622,2.3730741453$ $\mathrm{H}, 0,2.0571732916,1.1712042651,2.0436529924$ $\mathrm{H}, 0,2.1587046209,-0.3917198702,1.2835162377$

$\mathrm{HF}=-513.4704209$

Nimag $=1$

\section{CTS - B3LYP/6-31G(d)}

C, $\odot,-\odot .4419734585,0.2218351867,0.0731501404$ C, $0,-1.3299106205,1.1544173619,-0.4749096791$ C, $0,-2.6825832942,0.8405828884,-0.6087492895$ C, $0,-3.1556904974,-0.4058176426,-0.1955602865$ C, $0,-2.269080571,-1.3433758666,0.3414835141$ C, $0,-0.916278821,-1.0347332884,0.4732956355$ C, $0,0.9885626354,0.646730241,0.2257034903$ $0, \odot, 1.4941005929,1.6171768645,-\odot .3062403486$ $\mathrm{H}, 0,-0.942441858,2.1171182371,-0.7925925804$ $\mathrm{H}, 0,-3.3662209879,1.5695979572,-1.0356366088$ $\mathrm{H}, \odot,-4.2094899409,-0.6507255221,-0.2990421412$ $\mathrm{H}, \odot,-2.6303008097,-2.3225516743, \odot .6445500905$ $\mathrm{H}, 0,-0.216594291,-1.7813197514,0.8347169303$ $0,0,1.9321244563,-\odot .9853853352,-\odot .1736783914$ C, $0,2.8659575858,-0.809517912,-1.2047490042$ $\mathrm{H}, 0,3.8736419822,-1.1243777771,-0.8909848841$ $\mathrm{H}, 0,2.9208664448, \odot .2524072407,-1.5006357395$ $\mathrm{H}, 0,2.5875873897,-1.401129136,-2.0903314879$ $\mathrm{N}, 0,1.5772027739,0.299748167,1.6715692648$ $\mathrm{H}, \odot, 0.8946040031,0.0327547834,2.3812964018$ $\mathrm{H}, \odot, 2.1507740708,1.0853344949,1.9769014633$ $\mathrm{H}, 0,2.1333364329,-0.5504040661,1.1321364971$

\section{$C T S-B 3 L Y P / 6-31+G(d, p)$}

C, $-0.4416910003,0.2279272055,0.0444194698$ C, $-1.3147637329,0.9966017216,-0.7359038218$ C, $-2.6562979217,0.6275653398,-0.8592596176$ C, $-3.1342209224,-0.50906844,-0.2025068327$ C, $-2.2626153076,-1.2834403864,0.5708950669$ C, $-0.9208858885,-0.9201371331,0.6923894398$ C, $0.9787957623,0.701831165,0.1588107614$ $0,1.4762352813,1.5836946466,-0.5241594163$ $\mathrm{H},-0.9260675852,1.8748557632,-1.2406504669$ $\mathrm{H},-3.3263892016,1.2282039875,-1.4675597544$ $\mathrm{H},-4.1774217138,-0.7961946013,-0.2982901446$ $\mathrm{H},-2.6249800026,-2.1793118875,1.0667635107$ $\mathrm{H},-0.2356186029,-1.5549579256,1.244693078$ $0,1.9664673082,-0.9374959212,0.0417408461$ C, 2.8473422106, - - . 9727996903, -1.0544221815 H, 3.8607211121, - 1.2541412236, - - .7331126249 $\mathrm{H}, 2.905541777,0.0191704633,-1.5326071781$ H, 2.5076839235, - $1.7043583282,-1.8015982236$ $\mathrm{N}, 1.5450096952,0.6207406288,1.6391935285$ $\mathrm{H}, 0.8601976447,0.4525439079,2.3745315068$ $\mathrm{H}, 2.0870919779,1.4645099207,1.8160460129$ H, 2.1385728904, - ๑. 2759729742, 1.2702444414

$H F=-516.654607$

Nimag $=1$

\section{CTS - B3LYP /cc-pVTZ}

C, $\odot,-0.4340439943,0.2129249956,0.1447253693$ C, $\odot,-1.3007185664,1.2179932863,-0.2810444519$ C, $0,-2.647417952,0.9414736728,-0.4823868884$ C, $0,-3.1371462438,-0.3393584772,-0.2583117528$ C, $0,-2.2729214342,-1.347749912,0.1580484181$ C, $0,-0.926584346,-1.0758037854,0.3559249378$ C, $0,0.9966274639,0.5908903088,0.3676838795$ $0,0,1.5049285108,1.6338422148,0.0276485215$ $\mathrm{H}, 0,-0.9018914058,2.2075137467,-0.4531157139$ $\mathrm{H}, \odot,-3.3132231924,1.7268900833,-0.814379594$ $\mathrm{H}, 0,-4.1855381573,-0.5552289413,-0.4155661534$ $\mathrm{H}, 0,-2.6464014064,-2.3510720999,0.3136023017$ $\mathrm{H}, \odot,-0.247617771,-1.8741260158,0.6194588614$ $0,0,1.9284653911,-\odot .9392889495,-\odot .3761577988$ C, $0,2.7707879337,-0.5989933301,-1.4389887006$ $\mathrm{H}, 0,3.7818213528,-0.9957061189,-1.2875660515$ $\mathrm{H}, 0,2.8554236156,0.4931384871,-1.5353243939$ $\mathrm{H}, 0,2.3912472185,-0.998080129,-2.3870135292$ $\mathrm{N}, 0,1.6055016119,-0.0404515297,1.6947054476$ $\mathrm{H}, 0,0.9379456475,-0.4463001871,2.3411630913$ $\mathrm{H}, 0,2.1820214036,0.6645134415,2.1400010746$ $\mathrm{H}, 0,2.1490530314,-\odot .7530682341,1.0099713257$

$\mathrm{HF}=-516.8095965$

Nimag $=1$
$\mathrm{HF}=-516.6071616$

Nimag $=1$ 


\section{TS1 - HF/6-31G(d)}

C, $\odot,-0.2926808392,-0.0979287828,0.1372349958$ C, $0,-1.5716287823,0.4273131392,0.025042936$ C, $0,-2.675529534,-0.417115635,0.0097124347$ C, $0,-2.5050932787,-1.7884819349,0.107400931$ C, $\odot,-1.2254040171,-2.3189142798,0.2153272659$ C, $0,-0.1248400757,-1.4774828191,0.2266446588$ C, $0,0.9059222684,0.8431958437,0.1790810469$ $0,0,0.6521220646,2.1271805489,-0.0534494197$ $0,0,2.0004667113,0.3066166509,-0.4923401307$ C, $0,2.0139880139,0.5263464651,-1.8844793715$ $\mathrm{N}, \odot, 1.4350384983,1.041258595,1.6046830003$ $\mathrm{H}, 0,-1.6866769774,1.4910245217,-0.061363016$ $\mathrm{H}, \odot,-3.663686 \odot 492,-\odot .0022614851,-\odot .0833740227$ $\mathrm{H}, \odot,-3.3597130881,-2.4418105981, \odot .0942517222$ $\mathrm{H}, \odot,-1.0870688579,-3.3836228275,0.283257578$ $\mathrm{H}, 0,0.8660986476,-1.8900025596,0.2911922148$ $\mathrm{H}, 0,2.9413360579,0.1067054377,-2.2485998172$ $\mathrm{H}, 0,1.9700974752,1.5843276133,-2.1014958076$ $\mathrm{H}, 0,1.1805209002,0.0222568041,-2.3644004488$ $\mathrm{H}, 0,2.3787661025,0.7275176179,1.7344133248$ $\mathrm{H}, 0,0.8345928471,0.7159690535,2.3382301534$ $\mathrm{H}, \odot, 1.2113507149,2.1291166812,1.1556341349$

$\mathrm{HF}=-513.4810387$

Nimag=1

\section{TS1 - B3LYP/6-31G(d)}

C, $\odot,-\odot .3024900961,-0.0992167776,0.1272743139$ C, $0,-1.5953642054,0.4335420137,0.1092208918$ C, $0,-2.704687937,-0.4131447296,0.1102921619$ C, $0,-2.5285806005,-1.7982906169,0.1288802392$ C, $0,-1.2383839287,-2.3346627357,0.1407094041$ C, $\odot,-0.1289495932,-1.488917326,0.138363626$ $\mathrm{C}, \odot, 0.8858706441,0.8562338748,0.1522401187$ $0,0,0.6373730125,2.1343960594,-0.1366747091$ $0,0,2.041370324,0.2686359167,-\odot .4543158364$ C, $0,2.0965998962,0.4830998197,-1.8619195928$ $\mathrm{N}, 0,1.4203412867,1.114655877,1.6158368112$ $\mathrm{H}, 0,-1.7037012938,1.5128123274,0.0764591157$ $\mathrm{H}, 0,-3.7066097601,0.0081049579,0.0899580436$ $\mathrm{H}, \odot,-3.392394557,-2.458167556,0.1279572701$ $\mathrm{H}, 0,-1.0969141061,-3.4125060252,0.1460544299$ $\mathrm{H}, 0,0.8769202958,-1.8978996188,0.1319907798$ $\mathrm{H}, 0,3.0907159759,0.1626720455,-2.1834653164$ $\mathrm{H}, 0,1.9454605641,1.5416841697,-2.0929131339$ $\mathrm{H}, 0,1.3379689702,-0.1142159631,-2.3870903416$ $\mathrm{H}, 0,2.3538553424,0.736980026,1.7883886621$ $\mathrm{H}, \odot, 0.7693659167,0.8583977198,2.3592235722$ $\mathrm{H}, 0,1.2489118773,2.2034298343,1.0901366269$

\section{TS1 - B3LYP/6-31+G(d,p)}

C, $-0.2988190071,-0.0955720309,0.1365446637$ C, $-1.5909536086,0.4334983348,0.0450686977$ C, $-2.6999910985,-0.41748742,0.0277475635$ C, $-2.524214848,-1.8016635817,0.1046245996$ C, $-1.2335236191,-2.334440594,0.1918239085$ C, $-0.1248535688,-1.4854183257,0.2052638122$ C, $\odot .8944712663,0.8571665593,0.175904071$ $0,0.6447860742,2.1499277939,-0.0717753601$ $0,2.0350813516,0.2911789197,-0.4797520468$ C, $2.0381498998,0.504002855,-1.8935529245$ $\mathrm{N}, 1.4511702071,1.0684665536,1.6285770637$ $\mathrm{H},-1.7010828135,1.5105815723,-0.0253871531$ $\mathrm{H},-3.6997314962,0.0005647409,-0.0503232776$ $\mathrm{H},-3.3860836316,-2.4629001622,0.0907109974$ $\mathrm{H},-1.0912294775,-3.4103481574,0.2428791645$ $\mathrm{H}, 0.8793624598,-1.8957707942,0.2529692545$ H, 3. $0080452427,0.1529539441,-2.2509472711$ $\mathrm{H}, 1.9117953615,1.5666031187,-2.1196968571$ H, $1.2408621802,-0.0729346257,-2.3805731455$ $\mathrm{H}, 2.3973094461,0.7096502085,1.7599781297$ $\mathrm{H}, 0.827061568,0.7663529818,2.3755842427$ H, $1.2549678082,2.1666128087,1.1564393751$

$\mathrm{HF}=-516.6511761$

Nimag $=1$

\section{TS1 - B3LYP/cc-pVTZ}

$C, 0,-\odot .2984897754,-\odot .0956988753,0.1382498077$ C, $0,-1.5844617883,0.4308605517,0.0545059404$ C, $0,-2.6885047548,-0.4142315172,0.0350010088$ C, $0,-2.5145361924,-1.7915753656,0.1014162922$ C, $0,-1.2309623596,-2.3228926357,0.1810738238$ C, $0,-0.1275552589,-1.4793092194,0.1969010055$ C, $\odot, 0.8915566153,0.8562402008,0.1738923701$ $0, \odot, \odot .6434146013,2.1405699257,-0.0784601416$ $0,0,2.0302250986,0.2878561971,-0.4763952327$ C, $0,2.0329431748,0.4908401185,-1.8863708828$ $\mathrm{N}, 0,1.4439406448,1.0715884481,1.6314099467$ $\mathrm{H}, 0,-1.693857827,1.5040391195,-0.0090930353$ $\mathrm{H}, 0,-3.6842435005,0.0032019776,-0.0370330438$ $\mathrm{H}, 0,-3.3734923467,-2.4494131527,0.0853233669$ $\mathrm{H}, \mathrm{O},-1.0902672404,-3.394969236,0.2242418382$ $\mathrm{H}, \odot, 0.8720482784,-1.8899032348,0.2392663033$ $\mathrm{H}, 0,3.0097962528,0.1708392471,-2.2418660174$ $\mathrm{H}, 0,1.8748679138,1.543321574,-2.1204184472$ $\mathrm{H}, 0,1.2592542405,-\odot .1095886823,-2.3744779399$ $\mathrm{H}, 0,2.3871991461,0.7190507576,1.7622591451$ $\mathrm{H}, 0,0.8236035545,0.7636862626,2.3725690786$ $\mathrm{H}, 0,1.2384514515,2.1658077011,1.150185925$

$H F=-516.8041809$

$\mathrm{Nimag}=1$ 


\section{I1 - HF/6-31G(d)}

$C, \odot,-\odot .2663745181,-\odot .0585341275,0.1744768868$ C, $\odot,-1.5502006622,0.447806675,0.0344809333$ C, $0,-2.642558977,-0.4117737651,-0.0214749356$ C, $\odot,-2.4600631968,-1.7810620257,0.0591419619$ C, $0,-1.1755778161,-2.2939485543,0.1958127302$ C, $0,-0.0893599786,-1.4384216312,0.2529730505$ $\mathrm{C}, 0,0.9573681332,0.8557347142,0.300857148$ $0, \odot, \odot .5760236733,2.1318356987,-0.069607716$ $0,0,2.0160426815,0.3937367481,-0.47227775$ C, $0,1.8661036996,0.4268334461,-1.8700082583$ $\mathrm{N}, 0,1.5030141408,0.9129500028,1.6338661714$ $\mathrm{H}, \odot,-1.6971986499,1.5080663473,-0.0354834693$ $\mathrm{H}, 0,-3.6325558314,-0.005377034,-0.131442792$ $\mathrm{H}, 0,-3.3053945475,-2.444724856,0.0138929736$ $\mathrm{H}, 0,-1.0226916652,-3.3571897179,0.2547281383$ $\mathrm{H}, 0,0.9028180141,-1.8423102241,0.3487793981$ $\mathrm{H}, 0,2.7821976365,0.0246463058,-2.2792510177$ $\mathrm{H}, 0,1.7255284943,1.4402278109,-2.2238658314$ $\mathrm{H}, 0,1.0307271437,-0.1840401349,-2.194855163$ $\mathrm{H}, 0,2.1146950031,0.1406091054,1.7992224748$ $\mathrm{H}, 0,0.7789070708,0.9170273949,2.322958662$ $\mathrm{H}, \odot, 1.2293174036,2.7280270193,0.2657800538$

$\mathrm{HF}=-513.5563129$

Nimag $=\odot$

\section{I1 - B3LYP/6-31G(d)}

C, $\odot, \odot .2482028977,-\odot .195438219,-\odot .0329491275$ C, $0,1.0449259025,-0.6024631285,-1.1087407638$ C, $0,2.4376984767,-0.5581727095,-1.0120219445$ C, $0,3.0484762416,-0.1049725759,0.1576216839$ C, $0,2.2580002582,0.306290008,1.2337792281$ C, $\odot, 0.867940378,0.2604097228,1.138741048$ C, $0,-1.2801908066,-0.3072034123,-0.079245279$ $0,0,-1.6680329738,-0.38806077,-1.426442279$ $0,0,-1.9070014291,0.7714706919,0.5940366991$ C, $0,-1.7411642065,2.0473382706,-0.0145531888$ $\mathrm{N}, 0,-1.8122725041,-1.4777357151,0.6113748527$ $\mathrm{H}, \odot, \odot .5674984279,-\odot .944997489,-2.0200927746$ $\mathrm{H}, 0,3.0451850769,-0.8747606631,-1.8561145847$ $\mathrm{H}, 0,4.1322819562,-0.0689050947,0.2307176479$ $\mathrm{H}, \odot, 2.7247654313,0.6664639932,2.1470670666$ $\mathrm{H}, 0,0.2517413571,0.5885630658,1.9713200548$ $\mathrm{H}, \odot,-2.5672413211,-0.7594757471,-1.3928777056$ $\mathrm{H}, \Theta,-2.3473830558,2.7409931204,0.5728103007$ $\mathrm{H}, 0,-2.0912303608,2.0388289565,-1.0521384866$ $\mathrm{H}, 0,-0.6935631814,2.3748548679,0.0062633136$ $\mathrm{H}, 0,-1.8498176464,-1.2897182265,1.6109389634$ H, $\odot,-1.1893887818,-2.2697038904,0.465936936$

\section{I1-B3LYP/6-31+G(d,p)}

$C, 0,-\odot .3014294315,-\odot .0944170371,0.0951313104$ C, $0,-1.597206468,0.4344158892,0.1232764378$ C, $0,-2.703878189,-0.4180433869,0.1661511626$ C, $0,-2.522779075,-1.804040992,0.1745544526$ C, $0,-1.2298938391,-2.3357423284,0.1308288409$ C, $0,-0.1228853616,-1.484779478,0.0927167053$ C, $0,0.8917065648,0.8592788102,0.1369882274$ $0, \odot, \odot .6569197305,2.1456194213,-0.1570310667$ $0,0,2.0550059611,0.2764875311,-0.4514897755$ C, $0,2.1211171613,0.4695872444,-1.8680995319$ $\mathrm{N}, 0,1.3601167788,1.1146448333,1.6139342759$ $\mathrm{H}, 0,-1.7147535865,1.5130801437,0.1009452634$ $\mathrm{H}, 0,-3.7063341183,-0.000027058,0.1907409735$ $\mathrm{H}, 0,-3.3829268192,-2.4665569825,0.2104752029$ $\mathrm{H}, 0,-1.0838069413,-3.4123503308,0.1262814407$ $\mathrm{H}, 0,0.8829818936,-1.891139017,0.0535575324$ $\mathrm{H}, 0,3.0959141647,0.091317308,-2.1813534173$ $\mathrm{H}, 0,2.0273705913,1.5310186897,-2.1138594022$ $\mathrm{H}, 0,1.330377122,-0.0949475367,-2.3799377862$ $\mathrm{H}, 0,2.256965676,0.7065881449,1.8809643011$ $\mathrm{H}, 0,0.631874209,0.8864214421,2.2892612004$ $\mathrm{H}, \odot, 1.2376066514,2.1996734164,1.0842658665$

$H F=-516.6506662$

$\mathrm{Nimag}=0$

\section{I1- B3LYP /cc-pVTZ}

C, $0,0.2662732381,-0.1542483847,0.1000979188$ C, $0,1.056655944,-1.1319601508,-0.4986311201$ C, $\odot, 2.4347395093,-\odot .9619957727,-0.5944633912$ C, $0,3.0361080032,0.1861945502,-0.0967433333$ C, $0,2.2510818757,1.1686223045,0.4991712799$ C, $\odot, 0.8773966039, \odot .9981675276, \odot .5975968514$ C, $0,-1.2437112793,-0.3357478886,0.282236949$ $0,0,-1.6621062951,-1.3692420547,-0.5721341187$ $0,0,-1.9481327498,0.868053425,0.0337285021$ C, $\odot,-1.9145679769,1.3399538365,-1.3076472465$ $\mathrm{N}, 0,-1.6383151393,-0.6752575757,1.6407488332$ $\mathrm{H}, \odot, 0.5877039886,-2.0205512403,-0.8933166622$ $\mathrm{H}, 0,3.036619073,-1.7291919198,-1.063661661$ $\mathrm{H}, 0,4.1071235525,0.3182546257,-0.1739239858$ $\mathrm{H}, 0,2.7099919426,2.0693213926,0.8852727642$ $\mathrm{H}, 0,0.268085534,1.7673966144,1.0529624836$ $\mathrm{H}, 0,-2.5569583193,2.2169917016,-1.3342748738$ $\mathrm{H}, \mathrm{O},-2.2941304742,0.587888447,-1.9999667372$ $\mathrm{H}, 0,-\odot .9035901372,1.6257992523,-1.6094571444$ $\mathrm{H}, 0,-1.7628997679,0.1707503461,2.1817512918$ $\mathrm{H}, 0,-0.929539138,-1.2431902167,2.0864807179$ H, $0,-2.4961434276,-1.6810730672,-0.1995705416$

$\mathrm{HF}=-516.8596441$

Nimag $=\odot$ 


\section{TS2 -HF/6-31G(d)}

C, $\odot, 0.2755097416,-0.4521199036,0.0019969568$ C, $\odot, 0.9963495264,-0.58923634,-1.1756758672$ C, $0,2.3534474748,-0.2986680835,-1.1939615547$ C, $\odot, 2.9849872712,0.140752867,-0.042542789$ C, $\odot, 2.2597750929,0.2954290047,1.133010522$ C $, 0,0.909647647,-0.0009080768,1.1562690622$ C, $\odot,-1.1615817326,-0.8210685283,-0.0024477944$ $0,0,-1.8209771001,-0.8933902412,-1.1252838399$ $0,0,-2.0362602263,1.0729574264,-0.0243368095$ C, $0,-1.5711048099,2.3565044775,0.1071185962$ $\mathrm{N}, \odot,-1.6663467721,-1.4690481236,1.0276712535$ $\mathrm{H}, 0,0.4973007673,-0.9242466437,-2.0648810922$ $\mathrm{H}, 0,2.9107399816,-0.4128145526,-2.1061533039$ $\mathrm{H}, 0,4.0352209389, \odot .3715327509,-\odot .0589540626$ $\mathrm{H}, 0,2.7435283541,0.657372633,2.0222155986$ $\mathrm{H}, 0,0.3474697485,0.1641162721,2.0581517967$ $\mathrm{H}, 0,-2.1323804329,0.0817948403,-1.1195228325$ $\mathrm{H}, 0,-2.2874421637,3.0833608252,-0.285443877$ $\mathrm{H}, \odot,-0.6260359768,2.5250230802,-\odot .4232038774$ $\mathrm{H}, 0,-1.3991061781,2.6225793174,1.1558253666$ $\mathrm{H}, 0,-2.6484695213,-1.6334761145,1.0185089981$ H, $\odot,-1.2006807702$, - $1.4725555254,1.9041209151$

\section{TS2 - B3LYP/6-31G+(d,p)}

$C, \odot, 0.247985435,-0.4358298279,0.0160416326$ C, $0,0.9571462203,-0.4359550589,-1.1930746723$ C, $0,2.318079961,-0.125355597,-1.2084096159$ C, $\odot, 2.9800369477,0.1947402545,-0.0204457683$ C $, 0,2.2737594345,0.2092749682,1.1869082013$ C, $0,0.9152535212,-0.1037683341,1.2063609708$ C, $0,-1.1936085477,-0.7973216413,-0.0049108412$ $0,0,-1.8540536055,-0.8856821221,-1.1579828163$ $0,0,-2.0661407855,1.0339345388,0.0110567132$ C, $0,-1.4157567573,2.2729307923,0.0389388548$ $\mathrm{N}, 0,-1.6866401336,-1.5311359238,1.0127267849$ $\mathrm{H}, \odot, 0.4313141288,-0.6907415414,-2.1065460977$ $\mathrm{H}, 0,2.8611751099,-\odot .1354978957,-2.1487621187$ $\mathrm{H}, 0,4.0382743628,0.4384810205,-0.0336512705$ $\mathrm{H}, 0,2.7790111315,0.4739063144,2.11101984$ $\mathrm{H}, 0, \odot .3725082901,-0.0506453366,2.1463808707$ $\mathrm{H}, 0,-2.1766123831,0.1445312249,-1.0829257322$ $\mathrm{H}, 0,-2.1356224113,3.0807382062,-0.1679123407$ $\mathrm{H}, 0,-0.6032029775,2.3526862514,-0.7047345433$ $\mathrm{H}, 0,-0.9815293513,2.4720878641,1.032827195$ $\mathrm{H}, \mathrm{O},-2.6835991358,-1.6987634247,0.9997224393$ H, $\odot,-1.2310579887,-1.5271438854,1.912450517$

$\mathrm{HF}=-516.6599791$

Nimag $=1$

\section{TS2 - B3LYP /cc-pVTZ}

C, $\odot, 0.2466504544,-\odot .443422377,0.0196028867$ C, $0,0.9446026,-0.4219185979,-1.1876001664$ C, $0,2.2967990487,-0.1065071086,-1.2087973232$ C, $0,2.9625196654,0.1989268438,-0.0279693267$ C, $0,2.2683659157,0.1935759531,1.1780049506$ C $, 0,0.9189220898,-0.1248212198,1.2026488054$ C, $0,-1.1899846744,-0.8056136238,0.0025280047$ $0,0,-1.8496828671,-0.9048072398,-1.1420071236$ $0,0,-2.0428147246,1.0412856499,-0.0144938018$ C, $0,-1.4101965453,2.2793673224,0.0374265784$ $\mathrm{N}, 0,-1.6805649694,-1.5175151568,1.0280814941$ $\mathrm{H}, 0,0.4157917095,-0.6653327665,-2.0972154242$ $\mathrm{H}, 0,2.8307841638,-0.100131256,-2.1494324149$ $\mathrm{H}, 0,4.0150647977,0.4476197513,-0.0455338792$ $\mathrm{H}, 0,2.7773393481,0.4470367409,2.098113901$ $\mathrm{H}, 0,0.3854588964,-\odot .0865649847,2.14368942$ $\mathrm{H}, 0,-2.1482287782,0.1278025127,-1.099620603$ $\mathrm{H}, 0,-2.0990696401,3.0740578687,-0.2801890523$ $\mathrm{H}, 0,-0.5210397769,2.3456331606,-\odot .6090235187$ $\mathrm{H}, 0,-1.0908843055,2.5278461354,1.0602811275$ $\mathrm{H}, 0,-2.6727416939,-1.6878996559,1.0217070014$ H, $0,-1.2146105279,-1.5168118441,1.9175939292$

Nimag $=1$ 


\section{Catalyzed ammonolysis of methyl benzoate}

\section{$C^{\text {catal }}-B 3 L Y P / 6-31 G(d)$}

C, $\odot, \odot .7619068708, \odot .5696641371,-\odot .397799827$ C, $\odot,-0.5610758199,-0.191165807,-0.5094334226$ C, $0,-1.335275445,-0.6044112089,0.5825856488$ C, $\odot,-2.5951191525,-1.1742620839,0.3849449095$ $\mathrm{C}, 0,-3.0976144437,-1.3407854378,-0.9080305534$ C, $0,-2.3233917167,-0.9506214841,-2.0018984139$ C, $\odot,-1.0650446247,-0.3814324822,-1.8015803466$ $0,0,1.4629758611,0.7474130655,-1.4051758751$ $\mathrm{N}, 0,0.5084386234,1.8817232437,0.4291983755$ $\mathrm{H}, 0,-0.9483504605,-0.4963406618,1.5918803065$ $\mathrm{H}, 0,-3.1883481926,-1.488912374,1.2405303589$ $\mathrm{H}, \odot,-4.0844429149,-1.7714982591,-1.0606522082$ $\mathrm{H}, 0,-2.7038382834,-1.080420954,-3.0124924951$ $\mathrm{H}, 0,-0.4497423507,-0.0637431293,-2.6371265154$ $\mathrm{H}, \odot, 1.086831815,2.5604399387,-\odot .0703608459$ $\mathrm{H}, \odot,-\odot .4625105207,2.1687935106,0.2943394166$ $0,0,1.6238885739,-0.3489985668,0.8218703035$ C, $\odot, 2.9465924826,-0.6289442971,0.4212301878$ $\mathrm{H}, 0,3.6872293899,-0.0846587211,1.0385051439$ $\mathrm{H}, 0,3.0866277915,-\odot .318211876,-\odot .6224699656$ $\mathrm{H}, 0,3.1601230644,-1.7034112782,0.5114528394$ $\mathrm{N}, 0,1.4779112688,1.294195172,2.7457143252$ $\mathrm{H}, 0,2.3407356874,1.7604065429,3.0209100276$ $\mathrm{H}, \odot, \odot .8924830804,1.1324292891,3.5629360594$ $\mathrm{H}, \odot, 0.9391404167,1.7878987584,1.8212623902$ $\mathrm{H}, 0,1.6588278464,0.3902362976,2.1432320611$

$\mathrm{HF}=-573.1722359$

Nimag $=1$

\section{$T S 1^{\text {catal }}-B 3 L Y P / 6-31 G(d)$}

$C, 0,-0.5595071505, \odot .0242901883,-0.6775889811$ $0,0,0.0636506001,-0.3327116239,-1.9482756464$ $0,0,-1.696877201,-0.5592708567,-0.4324640523$ C, $0,0.0021252184,-1.7252414083,-2.215046117$ C, $0,0.4642033925,-0.0851503634,0.4679487339$ C, $\odot, 1.8455267698,-0.0586978928,0.2451882565$ C, $0,2.7359872423,-0.0974895814,1.3205393553$ C, $0,2.2541632858,-0.1440795735,2.6302347054$ C, $\odot, 0.875979536,-0.1698023248,2.8585797839$ C, $\odot,-0.0115043892,-0.1513045158,1.7826813213$ $\mathrm{H}, \odot,-1.0255435044,-2.0947111921,-2.140125634$ $\mathrm{H}, 0,0.3791170982,-1.8655890849,-3.2325602365$ $\mathrm{H}, 0,0.630193109,-2.3007598218,-1.5186609372$ $\mathrm{H}, 0,2.2130126384,-0.0173642088,-0.7756828049$ $\mathrm{H}, 0,3.8075642573,-0.0909648034,1.1352574615$ $\mathrm{H}, \odot, 2.9474149919,-0.1649979557,3.4674079666$ $\mathrm{H}, 0,0.4933131812,-0.2107362578,3.8757940765$ $\mathrm{H}, \odot,-1.0850968735,-0.1993330152,1.9368790791$ $\mathrm{N}, 0,-0.7388429559,1.5926355602,-0.9500161375$ $\mathrm{H}, 0,-0.3808125377,2.1197651024,-0.1541239272$ $\mathrm{H}, \odot,-\odot .1710512854,1.833641712,-1.7629423705$ $\mathrm{H}, 0,-2.1200901597,1.6989586819,-1.1222451427$ $\mathrm{N}, 0,-3.2417683376,1.2364976295,-1.2486812376$ $\mathrm{H}, \mathrm{O},-2.8157449276,0.2330016705,-1.1418150287$ $\mathrm{H}, \odot,-3.88770924,1.3794596412,-0.4733742738$ $\mathrm{H}, 0,-3.6963183168,1.4564098802,-2.1322313603$ $\mathrm{HF}=-573.1878537$ Nimag $=1$

\section{$T S 2^{\text {catal }}-B 3 L Y P / 6-31 G(d)$}

C, $0,0.9452439337,0.1985085332,-0.1272717752$ $0,0,1.5595824431,0.9119050081,0.7325362312$ $\mathrm{N}, 0,1.8289215766,-0.4952610573,-1.0720208073$ C, $\odot,-0.4733719728,-0.7081711584,1.7377775038$ C, $\odot,-\odot .25895983,-\odot .6017936303,0.3604222622$ C, $0,-1.132233968,-1.2622277023,-0.5151749919$ C, $\odot,-2.1916032215,-2.0219556882,-0.020642779$ C, $0,-2.3911658404,-2.1344210525,1.3582679156$ C, $0,-1.530313004,-1.4732672242,2.2351979643$ H, $0,2.4455790711,-1.0821603733,-0.5117787584$ $\mathrm{H}, 0,1.2998181735,-1.1076059783,-1.6899825047$ $\mathrm{H}, 0,0.2029984811,-0.179828205,2.4016490158$ $\mathrm{H}, 0,-1.0001723413,-1.1527624574,-1.5885904877$ $\mathrm{H}, 0,-2.8658867646,-2.5230576101,-0.7110147432$ $\mathrm{H}, 0,-3.2156715658,-2.729009038,1.743658279$ $\mathrm{H}, 0,-1.6827153351,-1.5505788336,3.3091019178$ $0,0,0.2158167305,1.2328308988,-1.3596844409$ C, $0,-0.7268999047,2.1226695334,-0.7914678113$ $\mathrm{H}, 0,-\odot .38002281,2.5025728516,0.1820996851$ $\mathrm{H}, 0,-1.6951452959,1.627560314,-0.6431273487$ $\mathrm{H}, 0,-0.8771658113,2.9725580268,-1.4719029116$ $\mathrm{N}, 0,2.5055366828,2.2795011921,-1.2780031928$ $\mathrm{H}, 0,2.4868871076,1.8448931516,-0.2993195403$ $\mathrm{H}, 0,2.6613813208,3.286120882,-1.2728551469$ $\mathrm{H}, 0,3.1854804396,1.7936303469,-1.8589748155$ $\mathrm{H}, 0,1.4460569719,1.9340490605,-1.5442586941$

Nimag $=1$ 
